15

\section{Feasibility of Using Wearable Sensors to Detect Agitation in Persons with Dementia}

Christianne Nesbitt, DNP, RN, GNP-BC, PMHNP-S'; Ajay Gupta, MS $S^{2}$; Kurt Maly, PhD ; Hamid Reza Okhravi, $\mathrm{MD}^{4}$; and Shubham Jain, $P h D^{5}$

${ }^{1}$ School of Nursing, Old Dominion University, Norfolk, VA \& Shenandoah University, Winchester, VA

${ }^{2}$ Department of Computer Science, Old Dominion University, Norfolk, VA

${ }^{3}$ Department of Computer Science, Old Dominion University, Norfolk, VA

${ }^{4}$ Glennan Center for Geriatrics and Gerontology, Eastern Virginia Medical School, Norfolk, VA

${ }^{5}$ Department of Computer Science, Old Dominion University, Norfolk, VA

ABSTRACT: BACKGROUND/METHOD: Wearable sensors have become increasingly accurate in measuring various aspects of health monitoring in humans. Persons with dementia (PWD) often experience problematic behavioral and psychological symptoms of dementia (BPSD). These behaviors can include kicking, hitting, biting, screaming, pushing and are stressful and dangerous for the PWD as well as for caregivers both formal paid caregivers and informal family caregivers. There are many proven methods to intervene during agitated behavior outburst and the earlier these methods are used the better the results. Such methods include redirection, one-on-one socialization, music therapy, pet assisted therapy, etc. These types of effective methods are preferred over routine or as needed medications to control the behaviors. The medications currently used have well documented adverse side effects, especially in aging adults.

This IRB approved study used a convenience sample of eight PWD who had a history of BPSD in an assisted living facility specializing in the care of PWD. We evaluated the use of off-the-shelf smart watch technology to measure limb movements, vocalizations, heart rate and location in a facility. The research goal was to determine the feasibility of using this technology to accurately measure patient data which in turn will allow clinicians to promptly detect agitation and provide early intervention. Output data from the watch was compared to data recorded by trained observers using the Cohen-Mansfield Agitation Inventory (CMAI). Data was collected in four-hour blocks of time over a two-day period.

RESULTS: All the participants wore the devices without difficulty. Observations were then compared to the information obtained from the smart watch technology. Limb movements and heart rate increases correlated well with observers' measurements of agitation. True positive measures were greater than $60 \%$ (data streams from devices correlated with observations). The voice measures of tone, volume and words used did not correlate well due to background noise in this communal environment.

CONCLUSIONS: Data streams did correlate with observations. This technology could be useful in quickly identifying, and potentially anticipate, agitation in PWD. Further research is pending that will fine tune our software developed to measure the data streams and enhance accuracy. Also, improvements are being made in the ability to use voice recognition technology to capture the vocalizations associated with agitation. This technology can be used to quickly identify and prevent escalation of some BPSD by allowing early application of nonpharmacologic methods to treat agitation. Further study will also evaluate the impact this may have on the quality of life for caregivers and PWD.

Funding Acknowledgements: Old Dominion University Office of Research, $\$ 2,500$

\section{7}

\section{D2 Receptor Partial Agonists in Dual Disorders:} Use of Aripiprazole in Psychotic Disorder and Comorbid Substance Use Disorder

Daniel Hernandez Huerta, $\mathrm{PhD}^{\prime}$; Elena Begoña Alonso Sanchez, $M D^{\prime}$; Carmen Aldara Carrajo Garcia, $M D^{\prime}$; and Rocio Torralba Viorreta, $M D^{\prime}$

Psychiatry Department, Ramon y Cajal University Hospital, Madrid, Spain

ABSTRACT: Objective: Dopaminergic mechanisms are involved in addiction but few effective drugs have been developed to treat it. Recent research has highlighted dopamine D2 receptor partial agonists, like aripiprazole, as a potential treatment for different types of substance dependence. In this study we investigate the use of both oral or long-acting injectable (LAI) aripiprazole in the treatment of dual disorders, specifically focusing on patients with psychotic disorder and comorbid substance use disorder.

METHOD: Retrospective mirror-image study was conducted at an adult psychiatry inpatient unit from a tertiary care center (Ramon y Cajal University Hospital, Madrid, Spain). Patients included were those with a comorbid psychotic disorder and substance-related disorders (excluding tobacco and caffeine), according to DSM-5 criteria, who started aripiprazole in 2017. The number of psychiatric acute inpatient admissions and psychiatric emergency room visits, six months before and after aripiprazole initiation, were obtained from patients' 